car que delírios bizarros e sintomas cenestopáticos parecem estar associados a pior prognóstico em pacientes psicóticos. ${ }^{5}$

Leonardo Baldaçara, Luciana P C Nóbrega, Andréa Freirias, Ana Paula Marques, Marsal Sanches Departamento de Psiquiatria, Faculdade de Ciências Médicas, Santa Casa de São Paulo, São Paulo (SP), Brasil

Financiamento: Inexistente

Conflito de interesses: Inexistente

Referências

1. Foulon C. Schizophrenia and eating disorders. Encephale. 2003;29(5):463-6.

2. Kato $\mathrm{S}$, Ishiguro $\mathrm{T}$. Clinical courses of hypochondriac-cenesthopatic symptoms in schizophrenia. Psychopathology. 1997;30(2):76-82.

3. Phillips KA. Psychosis in body dysmorphic disorder. J Psychiatr Res. 2004;38(1):63-72.

4. De Leon J, Bott A, Simpson GM. Dysmorphophobia: body dysmorphic disorder or delusional disorder, somatic subtype? Compr Psychiatry. 1989;30(6): 457-72.

5. Morozov PV. [Prognosis of juvenile schizophrenia with dysmorphophobic disorders (according to catamnestic findings]. Zh Nevropatol Psikhiatr Im S S Korsakova. 1976;76(9):1358-66. Russian.

\section{"Qualidade de vida em irmãos de autistas" - I}

"Quality of life in siblings of autistic patients" - I

\section{Sr. Editor,}

Consideramos relevante o artigo "Qualidade de vida em irmãos de autistas", pois tem havido um consenso acerca da necessidade de desenvolvermos melhores instrumentos de avaliação. ${ }^{1}$ Qualidade de vida (QV) é uma variável interessante para os estudos em populações especiais e/ou submetidas a determinadas intervenções. Sua definição engloba diferentes aspectos como estilo de vida, comunidade e vida familiar. QV consiste na possessão dos recursos necessários para a satisfação das necessidades e desejos individuais, participação em atividades que permitem o desenvolvimento pessoal, a auto-realização e uma comparação satisfatória entre si mesmo e os outros. ${ }^{2}$

Face a sua ampla definição, observa-se que os trabalhos que avaliam QV devem delimitar claramente as relações que estão sendo estudadas. ${ }^{3}$ A necessidade de uma clara definição do objeto de estudo é um dos pontos que gostaríamos de comentar acerca do estudo publicado.

O AUQEI (Autoquestionnaire Qualité de Vie Enfant Imagé) é um instrumento validado e reconhecidamente eficaz na avaliação de QV em crianças e adolescentes. Porém, os autores do instrumento sugerem que os dados adquiridos devem ser complementados com outros questionários a fim de se obter informações mais completas e precisas. ${ }^{4} \mathrm{~A}$ hipótese apresentada no artigo da RBP foi de uma pior QV em irmãos de autistas, possivelmente relacionada à presença desse irmão. Nossa preocupação está na impossibilidade do estabelecimento de relações causais quando se utiliza esse questionário sem controle de outras variáveis como, por exemplo, a presença de vulnerabilidade genética ou comorbidades nesses irmãos. Além disso, QV pode ser influenciada pela dinâmica da família em seus vários aspectos (relacionamento marital, cuidado filial, continência parental, situação econômica para o custeio dos filhos, etc.).
Vários pontos podem ser reafirmados e destacados neste artigo, sua originalidade e a importância em relação à promoção de saúde mental, com isso contribuindo para uma meIhora de bem estar. Porém, muitos dos problemas encontrados em relação à aceitação e inclusão de crianças autistas são devidos a posições pré-concebidas em relação a eles, suas possibilidades e suas famílias. Um dos principais preconceitos encontrados na literatura surgiu com Leo Kanner, sugerindo como possível causa do distúrbio a presença de "mães geladeira". Esta conclusão foi decorrência do viés metodológico (no caso, um viés na origem da amostra) e pelos a priori teóricos que predominavam na época (o modelo psicodinâmico).

Uma consideração metodológica relacionada ao artigo seria a escolha pelo grupo controle. Já na década de 70, Rutter sugere que o pareamento mais eficaz no estudo populacional de autistas seria crianças com comprometimento da comunicação - como afasias receptivas/expressivas - e não crianças com problemas articulatórios. ${ }^{5}$

É imprescindível que continuemos a estudar os transtornos invasivos do desenvolvimento, principalmente na nossa população que conta com poucos programas de apoio. Esse estudo vem contribuir para o preenchimento de uma parte dessa lacuna, buscando, com isso, minimizar os estigmas e melhorar a QV dos portadores de autismo.

Rosane Lowenthal, Maria Lucila Ribeiro Campos, Cristiane Amorosino, Antonia Gomila, Maria Eloísa Fama D'Antino

Programa de Mestrado em Distúrbios do Desenvolvimento, Universidade Presbiteriana Mackenzie, São Paulo (SP), Brasil

Financiamento: Inexistente

Conflito de interesses: Inexistente

Referências

1. Marciano ARF, Scheuer $\mathrm{Cl}$. Quality of life in siblings of autistic patients. Rev Bras Psiquiatr. 2005;27(1):67-9.

2. Shin DC, Johnson DM. Avowed happiness as an overall assessment of the quality of life. Social Indicators Res. 1978;5:475-92 apud Assumpção Junior FB, Kuczynski E, Spovieri MH, Aranha EMG. Escala de avaliação de qualidade de vida. Arq Neuropsiquiatr. 2000;58(1):119-27.

3. Gill TM, Feinstein AR. A critical appraisal of the quality of quality of life measurements. JAMA. 1994;272(8):619-26.

4. Assumpção Junior FB, Kuczynski E, Sprovieri MH, Aranha EMG. [Quality of life evaluation scale (AUQUEI - Autoquestionnaire Qualite de Vie Enfant). Validity and realibility of a quality of life for children 4 to 12 years -old]. Arq Neuropsquiatr. 2000;58(1):119-27. Portuguese.

5. Cantwell DP, Baker L, Rutter M. Families of autistic and dysphasic children. II. Mothers'speech to the children. J Autism Child Schizophr. 1977;7(4):313-27. 